International Journal of Engineering \& Technology, $7(1.5)(2018)$ 234-236
International Journal of Engineering \& Technology
SPC
Website: www.sciencepubco.com/index.php/IJET
Research paper

\title{
Cantillever based MEMS pressure sensor
}

\author{
M Siva Kumar*, K.Srinivas Rao, Sanath Kumar Tulasi, G R K Prasad, K Hari Kishore \\ Department of ECE, Koneru Lakshmiah Educational Foundation, Vaddeswaram, Guntur, Andhra Pradesh, India 522502 \\ *Corresponding author E-mail: siva4580@ kluniversity.in
}

\begin{abstract}
Objective: We are analyzing the different piezoelectric materials which are best sutable combination for the design of pressure sensors used for the production of voltage. Our main theme is to find which combination gives high voltage.

Methods:We are incorporating different layers on the cantilever beam such as piezoelectric material ( $\mathrm{PZT}, \mathrm{BaTiO})$, electrode( $\mathrm{Pt}, \mathrm{Al})$, insulator $\left(\mathrm{Si}_{3} \mathrm{~N}_{4}\right)$ on siliconwafer $\left(\mathrm{SiO}_{2}\right)$. We had used these materials for MEMS pressure sensor utilizing diverse [different] Piezoelectric materials The relative study by simulation can be utilized to give the rules to a configuration and streamlining of execution of the distinctive piezoelectric micro cantilever pressure sensors.

Findings:. We have designed, displayed and simulated our module in COMSOL software taking into account limited component strategy [FEM]. The structure made up of silicon on piezoelectric micro cantilever with cantilever beam. In reenactment [simulation] we have concentrated on varying so as to displacement and voltage connected pressure. It is observed that the induced voltage is practically straight [linear] with the applied force

Improvement:From this design we analysed that at particular dimensions of materialswithcombinationofPZTand Al gives best voltage than remaining combinations like PZT and Pt.
\end{abstract}

Keywords:Cantilever beam, MEMS [micro electro mechanical systems], Piezoelectric materials, Pressure based Sensor, Finite Element method [FEM].

\section{Introduction}

The MEMS engineering [Micro Electro mechanical Systems] need pulled done various examiners since late decades especially in micro sensors Furthermore actuators. Previously, MEMS weight sensors need aid a standout amongst the vital sensors around them. These weight sensors have been depended once different physical properties in piezoresistive, electric, capacitive. However, as Compared will different MEMS advances, piezoelectric MEMS prescribe uncommon prizes1. Smits Furthermore Choi introduced electromechanical trademark of a heterogeneous piezoelectric binge relies once different electrical Furthermore mechanical (physical) limit conditions: A mechanical minute to the conclusion of the bender, An weight associated inverse of the tip of the binge Also a uniform load associated again the entirety period of the binge. Zhang and Sun 2 portrayed the association between minimum observable energy inclinations Furthermore level estimations clinched alongside noncontact examining energy microscopy using piezoelectric micro cantilever.

FEA [finite component analysis] the usage of COMSOL Multiphysics carry been utilized within this fill in to interrupt down those outcomes of the multi-layer cantilever structures using piezoelectric materials (PZT, BaTio3, Zns). That configuration of a shape is used already, made $\mathrm{SiO} 2$-based cantilevers3. using this structure those features from claiming piezoelectric material want been depicted or placed. those re-enactment comes about display the ones uprooting furthermore electric powered potential are incremented particularly Likewise joined weight additions. on this work, correlations from claiming re-enactment from claiming extraordinary piezoelectric materials (PZT, BaTiO3 and $\mathrm{ZnS}$ ) have been completed the use of equal structure. The to be had setup what is more operational measures would cartoon out on this paper.

\section{Theory}

"Piezoelectricity" is clarified as the potential of specific materials to create electrical rate due to mechanical deformation. There are not any sure popular strategies for to compute the electromechanical piezoelectric parameters. records approximately the layers in multilayer cantilever is regarded in table 14.

on this design, two specific methodologies are determined the elements of the multi-layer cantilevers beam. The most important method clarifies theoretical dating among surface stress (strain) and tip elimination at the upper layer of the micro cantilever and the evaluation among the piezoelectric tip displacement and the affected stage voltage is cleared. Piezoelectric materials deform then electric charge totals on repudiating surfaces and makes a voltage whilst strained by using an out of doors force5. that is a right away result of the immutable (perpetual) dipole nature of those materials. right while differential floor tension is at the top layer of the cantilever, the tip displacement (z) finished with the aid of the differential floor pressure(s) may be made as.

$Z=\frac{3(1-v) l}{t^{\wedge} 2 e} \Delta s$

Here $l$ is the cantilever length, $t$ is the thickness of cantileve, $v$ is the Poisson proportion, $\Delta \mathrm{S}$ is the differential surface area of the stress (pressure), and $e$ is the Young's modulus. 
When the pressure applied on the cantilever beam and the beam displaces from that force. Due to this the voltage is generated and it is calculated from the given equation 2 .

$V=\frac{t^{2} e_{e}}{3 \mathrm{~d} l^{2} \varepsilon_{p}} Z$

Revising the comparision (2) utilizing the mathematical statement (1) and composed as equation 3.

$V=\frac{e_{e}(1-v)}{\mathrm{d} e_{p} \epsilon} \Delta S$

Here $V$ is the voltage created or connected from the piezoelectric substrate, Young's modulus $e_{p}$ (piezoelectric material), $e_{e}$ is the Young's modulus of elasticity elastic materials and $d$ is the piezoelectric consistent of the piezo electric -material which we are using.

In the second technique, $\operatorname{FEM}^{6}($ Finite Element Method recreations or simulation) utilizing COMSOL with solid mechanics and electric resistance have been executed on multilayer cantilevers ${ }^{7}$.

\section{Design and simulation}

Inside the present take a look at our predominant intension is to find FEA [finite element analysis] COMSOL Multiphysics program is used for simulation of our shape. COMSOL software program device is a limited component analyzer, solver and Simulation programming software. This FEA [finite element analysis] software has bundles for distinctive cloth of science and physics designing programs. This FEA[Finite element analysis] software has a MEMS bundle. shape 1 has platinum $(\mathrm{Pt})$ fabric as anode and in shape 2 has aluminum ( $\mathrm{Al})$ steel as anode terminal. Measurements and fabric of structure 1,2 has been displayed in desk 2, 3. Structure2 aspect image of the layout has been seemed in figure 1 and parent 2 shows the 3-d view of the layout in COMSOL software. on this re-enactment(simulation) we've got few assumptions as follow

1. every and every piezoelectric layer have starting now been captivated(polarized) and the voltage received may not alternate the polarization fabric.

2. The cantilever bendy stop is depicted because the starting place of the X-bearing. this is except sturdy with the cutoff situations three. Settled primary is made in $\mathrm{Y}-\mathrm{Z}$ bearings at inception aspect. 4. every one of the houses of materials are taken as default residences of era (reproduction) programming on this reenactment (simulation) we've some assumptions. these are taking after presumption.

Fig 1.Shows Different layers side view of the our layout inside the software for second view.

Fig 2.Shows 3D view of multilayer cantilever beam in COMSOL software

\section{Results and discussion}

Reproduction about this fill in would done Eventually Tom's perusing piezoelectric gadgets8 (materials) model about COMSOL Multiphysics. This propagation cost (simulation) investigations each a standout amongst the parameters distinguished for piezoelectric materials. Piezoelectric more diminutive scale (micro) cantilever structure may be produced using 3d robust parts. That structure will be squashed which comprises from claiming 50,000 will 90,000 segments. Stationary sort investigation may be concluded for Recreation outcomes. Firstly Recreation outcomes are accomplished for structure 1(table2). Surface weight $(\mathrm{N} / \mathrm{m} 2)$ is set in $X$ What's more $Y$ heading What's more twisting for multilayer cantilever will be along On Z course. That relocation of particular case conclusion multilayer cantilever will be particularly relative on joined surface pressure, scientific comparison (1).

Figure 3 gives the information of the displacement when we applied the load the tip of the cantilever beam of the flexible end has the maximum displacement up to $(3.8 \mathrm{um})$ other end is fixed. Mainly we had observed that displacement changes with respect to the applied pressure in a linear manner.Figure4shows that it is arc length graph and from that we can say that displacement increasing with respect to the length of the cantilever beam when $5\left(\mathrm{Nm}^{\wedge} 2\right)$ pressure applied.

Figure 5, 6shows the linear increment of displacement and electric potential ${ }^{9}$ with respect to the applied pressure $5 \mathrm{~N} / \mathrm{m}^{\wedge} 2$. From above simulated results it knows that structure 2 giving best results compared to structure1. Pt (platinum) act like as an electrode in the structure 1 and in the structure 2 have $\mathrm{Al}$ (aluminum) as an electrode. Compared to the platinum $(\mathrm{Pt})$ with aluminum $(\mathrm{Al})$, aluminumhave low values of Poisson ratio and Young's modulus. From the Figure7, we abtained that Zinc sulfide having better displacement compared to barium titanate. In the Figure7 series1 for Zinc sulfide and series-2 give the information about Barium titanate, the below graph is showing that increment of displacement with respect to applied pressure increases.

\section{Conclusion}

In this paper we mentioned about piezo electric materials[PZT,Batio3,Zns] and how the properties of the material effecting to pressure sensor application. In the COMSOL software we had designed the structure which is having multi layers and the multi layers we used the different piezo materials by using the FEA [finite element analysis] and based on the simulation results among the three piezo materials as above mentioned [PZT,Batio3,Zns],PZT is giving best results like more displacement and electric potential. Coming to the electrodes $\mathrm{Al}$ and $\mathrm{Pt}, \mathrm{AL}$ is giving the best results compare to the $\mathrm{Pt}$ the values of Poisson ratio and Young modulus is very less. From this analysis we observed that electric potential range is (1-10) mv and by getting from this combination of $\mathrm{Al}$ (aluminum) and PZT (lead zirconium titanate) and while other combinations structures were not able to get above

\section{References}

[1] V. Khanaa1 and K. P. Thooyamani, Design and Analysis of an Integrated MemsScience and Technology , Indian Journal Of Science And Technology, Vol8(32), 10.17485/ijst/2015/v8i32/87626

[2] ZhangX.D., SunC.T. Minimum detectable force gradients of piezoelectric microcantilever, Journal of Micromechanics and Microengineering. 1995,5,pp.231-236.

[3] Behrens I., Doering L. and Peiner E. (2003). Piezoresistive Cantilever as Portable Micro Force Calibration Standard.Journal of Micromechanical Microengineering, Vol. 13, pp. 171-177.

[4] J. John Livingston and M. Hemalatha,chargingAn electronic Gadget using piezoelectricity, Indian Journal of Science and Technology, Vol 7(7), 945-948, July 2014.

[5] Wei Zhou, Abdul Khaliq, Yanjun Tang ,HaifengJi, Rastko R. Selmic. Simulation and design of piezoelectric microcantilever chemical sensors, Sensors and actuators A: Physical. 2005, 125(1),pp.69-75.

[6] G. Wu, H. Ji, K. Hansen, T. Thundat, R. Datar, R. Cote, M.F. Hagan,A.K. Chakraborty, A. Majumdar, Origin of nanomechanicalcantilevermotion generated from biomolecular interactions, Proc. Natl.Acad. Sci. USA 4 (2001) 1560-1564.

[7] Bhatti M. A., Lee C. X., Lee Y. Z. and Ahmed N. A. (2007). Design and Finite Element Analysis of Piezoresistive Cantilever with Stress Concentration Holes. 2nd IEEE Conference on Industrial Electronics and Applications.

[8] Liu M., Cui T., Dong W., Cuil Y., Wang J., Du L., Wang L. Piezoelectric microcantilevers with two with two PZT thin-film elements for microsensorsand microactuators, in Proceedings of the 1st IEEE International Conference on Nano/Micro Engineered and Molecular Systems, Zhuhai, China, 2006. 
[9] Dr. Seetaiah Kilaru, Hari Kishore K, Sravani T, Anvesh Chowdary L, Balaji T "Review and Analysis of Promising Technologies with Respect to fifth Generation Networks", 2014 First International Conference on Networks \& Soft Computing, ISSN:978-1-47993486-7/14,pp.270-273,August2014.

[10] Meka Bharadwaj, Hari Kishore "Enhanced Launch-Off-Capture Testing Using BIST Designs" Journal of Engineering and Applied Sciences, ISSN No: 1816-949X, Vol No.12, Issue No.3, page: 636643, April 2017.

[11] N Bala Dastagiri, Kakarla Hari Kishore "Reduction of Kickback Noise in Latched Comparators for Cardiac IMDs" Indian Journal of Science and Technology, ISSN No: 0974-6846, Vol No.9, Issue No.43, Page: 1-6, November 2016.

[12] A Murali, K Hari Kishore, D Venkat Reddy "Integrating FPGAs with Trigger Circuitry Core System Insertions for Observability in Debugging Process" Journal of Engineering and Applied Sciences, ISSN No: 1816-949X, Vol No.11, Issue No.12, page: 2643-2650, December 2016.

[13] Mahesh Mudavath, K Hari Kishore "Design of CMOS RF FrontEnd of Low Noise Amplifier for LTE System Applications Integrating FPGAs" Asian Journal of Information Technology, ISSN No: 1682-3915, Vol No.15, Issue No.20, page: 4040-4047, December 2016.

[14] P Bala Gopal, K Hari Kishore, B.Praveen Kittu "An FPGA Implementation of On Chip UART Testing with BIST Techniques", International Journal of Applied Engineering Research, ISSN 0973-4562, Volume 10, Number 14 , pp. $34047-$ 34051, August 2015

[15] S Nazeer Hussain, K Hari Kishore "Computational Optimization of Placement and Routing using Genetic Algorithm" Indian Journal of Science and Technology, ISSN No: 0974-6846, Vol No.9, Issue No.47, page: 1-4, December 2016.

[16] N Bala Gopal, K Hari Kishore "Analysis of Low Power Low Kickback Noise in Dynamic Comparators in Pacemakers" Indian Journal of Science and Technology, ISSN No: 0974-6846, Vol No.9, Issue No.44, page: 1-4, November 2016.

[17] T. Padmapriya and V. Saminadan, "Distributed Load Balancing for Multiuser Multi-class Traffic in MIMO LTE-Advanced Networks", Research Journal of Applied Sciences, Engineering and Technology (RJASET) - Maxwell Scientific Organization , ISSN: 2040-7459; e-ISSN: 2040-7467, vol.12, no.8, pp:813-822, April 2016

[18] S.V.Manikanthan and T.Padmapriya "Recent Trends In M2m Communications In 4g Networks And Evolution Towards 5g", International Journal of Pure and Applied Mathematics, ISSN NO:1314-3395, Vol-115, Issue -8, Sep 2017.

[19] Rajesh, M., and J. M. Gnanasekar. \&quot;Path observation-based physical routing protocol for wireless ad hoc networks.\&quot; International Journal of Wireless and Mobile Computing 11.3 (2016): 244-257.

[20] M. Rajesh, Manikanthan, "GET-UP-AND-GO EFFICIENT MEMETIC ALGORITHM BASED AMALGAM ROUTING PROTOCOL", International Journal of Pure and Applied Mathematics, ISSN NO:1314-3395, Vol-116, No. 21, Oct 2017. 